\title{
Droege, Peter; Knieling, Jörg (Hrsg.) (2017): Regenerative Räume. Leitbilder und Praktiken nachhaltiger Raumentwicklung
}

\author{
München: oekom Verlag. 388 S., 72 Abb., 12 Tab.
}

\author{
Sebastian Heilmann ${ }^{1}$ (D) \\ Online publiziert: 12. Februar 2018 \\ ๑) Springer-Verlag GmbH Deutschland, ein Teil von Springer Nature 2018
}

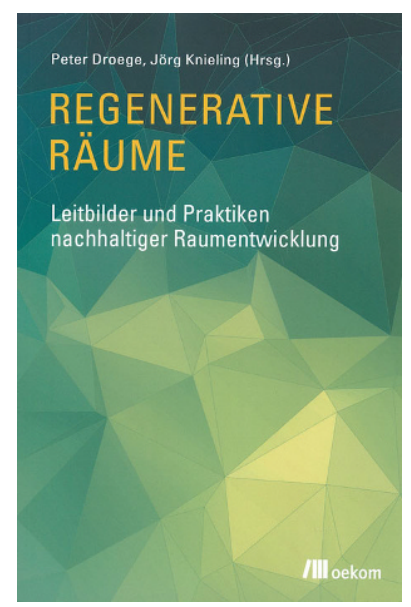

Peter Droege und Jörg Knieling widmen sich in dem von ihnen herausgegebenen Sammelband dem großen Feld der nachhaltigen Raumentwicklung. Das Buch fasst Beiträge der 2013er- und 2014er-Tagung des seit 2007 bestehenden internationalen Doktorandenkollegs „Nachhaltige Raumentwicklung“ (DokoNaRa) der Universitäten Innsbruck, Kassel, Liechtenstein und der HafenCity Universität Hamburg zusammen. Während die Tagung 2013 in Hamburg zum Thema ,Visionen und Leitbilder für eine nachhaltige Stadt- und Regionalentwicklung" stattfand, behandelte die 2014er-Tagung in Liechtenstein das Thema „Regenerative Region: erneuerbar, ressourcenschonend und resilient". Der gewählte Titel des nunmehr vorliegenden Doppelbandes vermag es gut, die inhaltliche Breite abzudecken hier liegt jedoch auch die Krux einer solchen Zusammen-

Sebastian Heilmann

sebastian.heilmann@uni.leuphana.de

1 Leuphana Universität Lüneburg, Fakultät

Nachhaltigkeit, Institut für Nachhaltigkeitssteuerung,

Universitätsallee 1, 21335 Lüneburg, Deutschland

stellung. Gelingt es den Herausgebern, die Beiträge so zu setzen, dass sich ein anregendes Studium des aktuellen Arbeitsstandes von Dissertationen aus dem deutschsprachigen Raum aus den Bereichen der nachhaltigen Stadt-, Regionalund Raumentwicklung ermöglicht? Es lässt sich konstatieren, dass „Regenerative Räume“ dazu in jedem Fall in der Lage ist. Bringen die Leser die Bereitschaft auf, sich auf eine interdisziplinäre Auseinandersetzung einzulassen - wobei ihnen kaum alle Themen bekannt sein dürften -, dann ist genau die vorliegende Vielfältigkeit auch die Stärke des Sammelbandes, denn auch in der Tiefe wissen die einzelnen Beiträge mit aktuellen und zukunftsorientierten Fragestellungen, Forschungskonzeptionen und großer Methodenvielfalt $\mathrm{zu}$ begeistern.

Gegliedert sind die 21 Beiträge in sieben Kapitel mit jeweils durchschnittlich drei Beiträgen. Die Autoren interessieren sich für unterschiedliche Orte, von Hamburg über Kopenhagen bis Schanghai. Poststrukturalistische Diskursanalysen finden sich ebenso wie der Aufbau quantitativer Bewertungsschemata und Dokumentenanalysen. Dabei lässt sich feststellen, dass die Autoren insbesondere aktuell (noch) marginalisierte Perspektiven und Fragen in den Vordergrund rücken. Dies zeigt sich an der Auswahl sowohl der theoretischen Grundlegungen der Arbeiten als auch an behandelten Inhalten. Neue Verhandlungsformen gesellschaftlichen Zusammenlebens werden angeregt und vermeintlich klare Strukturen als hybride Verhältnisse neu beschrieben.

\section{Kapitel "Leitende Bilder"}

Jörg Knieling und Martin Krekeler diskutieren „Leitbilder als Instrument für eine nachhaltige Raumentwicklung“. Sie stellen die Vielfältigkeit des Leitbildbegriffs heraus, fokussieren die Leitbilder der zweiten Generation, die kooperativ, reflexiv und innovativ ausgerichtet, maßgeblich zu ei- 
ner nachhaltigen Raumentwicklung beitragen können. Ulf Hahne und Simone Markert stellen in ihrer fünf Gemeinden umfassenden Fallstudie „Leitbild Bürgerengagement und die Herausforderungen des demographischen Wandels“ fest, dass bürgerschaftliches Engagement ein wichtiger Faktor im Dorf ist, jedoch nicht zur Sicherung von Lebensqualität instrumentalisiert werden darf. Peter Droege appelliert in „Beyond Smart: Intelligent Cities and Terrestrial Habitability“ für eine globale Energiewende als friedenspolitische Notwendigkeit. Frank Schulz beschäftigt sich mit ,Stadtgestalt im Stadtumbau. Potenziale für eine nachhaltige Stadtentwicklung“. Aus der Perspektive der Chartas von Aalborg und Leipzig fragt der Autor nach den Möglichkeiten der Umgestaltung der Städtebauförderung im Sinne einer verstärkten Berücksichtigung gestalterischer Perspektiven.

\section{Kapitel „Energie und Raum"}

Jonas Abraham und Thomas Köhler stellen ihr Modell „Energiewende-Städte/Transition Towns. Ein Beteiligungsmodell für resilienz- und suffizienzbasierte Leitbilder und Visionen in der Stadtentwicklung" vor. Nach einer Einordnung der Überlegungen zu ,Transition' skizzieren sie ihre Fallstudie zu einer weiterführenden Stadtentwicklungsstrategie in Hannover. Susanna Erker geht in ihrem Beitrag auf die „Räumliche Resilienz im Hinblick auf Energiekrisen“ ein. Aufbauend auf dem österreichischen Ansatz von Energieraumplanung stellt die Autorin ein normatives Konzept räumlicher Resilienz auf, welches - Raumtypen unterscheidend - einen wichtigen Beitrag zur Weiterentwicklung von Nachhaltigkeit leistet. Simone Markert sieht „Alternative Wege in der Stadtentwicklung. Slow Cities und Transition Towns als Anstöße für eine wachstumsunabhängige Stadt?". Sie arbeitet die Wachstumsabhängigkeit heutiger Städte heraus und sieht in der Entschleunigung Potenziale zur Umsteuerung in Richtung Nachhaltigkeit.

\section{Kapitel „Frei-Räume“}

Rieke Hansen beschäftigt sich mit der Frage „Grüne Infrastruktur für Städte - ein neuer Begriff für bereits bekannte Planungsstrategien oder zukunftsweisendes Konzept?“. Sie zeigt, dass die Prinzipien Konnektivität, Multifunktionalität und Transdisziplinarität der grünen Infrastruktur in Planungsdokumenten bereits nachweisbar sind. Christoph Herrmann schlägt in seinem Beitrag „Konfektionierter Raum: Handlungsbeschränkung oder Handlungsspielräume in urbanen Freiräumen?", aufbauend auf der Untersuchung von Freiraumproduktionen, eine dynamische und nachhaltige Entwicklung von Räumen vor, gemeinsam gestaltet - von Planern und Nutzern. Philip Grell berichtet aus „Innerstädtisches: Eine kultur- und sprachgeschichtliche Untersuchung am Beispiel ,innerstädtischer' Orte in Schanghai" und zeigt auf, dass Sprachanalyse ein Teil von Stadtforschung sein sollte, so Missverständnissen vorgebeugt werden kann und dies auch interdisziplinäres Planen der Städte zukünftig erleichtern könnte.

\section{Kapitel „Regionale Räume“}

Sylwia Patron forscht zu „Die Regionalpolitik der polnischen Region Masowien im Spannungsfeld von Innovation und Nachhaltigkeit", wobei sie aufzeigt, dass Innovationsförderung und nachhaltige Regionalentwicklung integrativ und nicht als komplementäre Strategien behandelt werden dürfen. Clément Guasco schreibt über „The TEN-T policy and the Fehmarn Belt Tunnel: Impact on regional development between Öresund and Hamburg“; dabei regt er, aufbauend auf Untersuchungen zum Ärmelkanaltunnel, die Integration der Region in die europäische Verkehrspolitik an.

\section{Kapitel „Werte und Raum"}

Magda Rich berichtet aus „The ,Healing City“ - A literature review exploring the introduction of therapeutic horticulture in urban planning in developed countries". Die Autorin zeigt die Potenziale für psychische Gesundheitsförderung im urbanen Raum am Beispiel von Kleingärten für Demenzkranke auf. Vera Kaps widmet sich dem „Lebensstil Kalkbreite: Gebaute Nachhaltigkeit als identitätsstiftendes Merkmal am Beispiel des Wohn- und Gewerbebaus Kalkbreite in Zürich“; sie schildert, wie es mit einem genossenschaftlichen Bauprojekt möglich ist, Nachhaltigkeit baulich-räumlich umzusetzen. In „Räumliche Wandlungsprozesse in ländlich bezeichneten Regionen im Kontext des gesellschaftlichen Wertewandels" zeigt sich Simone Linke hoffnungsvoll, dass in der Hybridisierung und Pluralisierung gute Chancen für eine nachhaltige Entwicklung sogenannter ländlicher Räume liegen.

\section{Kapitel „Fair Play und Space“}

Armin Kratzer präsentiert in „Of Bioneers and Creative Destruction? Linking Entrepreneurship and Transitions to Sustainable Development in Biosphere Reserves" ein Forschungskonzept, um den Beitrag von regionalen Akteuren herauszustellen, die sich für nachhaltige Regionalentwicklung stark machen. Daniel Magalhães fragt: „Does Fairtrade certification enhance small farmers' resilience and regional development?" und stellt fest, dass dies nur zum Teil der 
Fall ist, häufig doch eher größere Genossenschaften einen Vorteil durch Teilnahme am Fairtrade-Markt genießen. Clarissa Reikersdorfer nähert sich „Foundations of a Theory of Sustainability in Architecture and Planning" und erkennt, dass Nachhaltigkeitszertifizierungen über die unmittelbare Gebäudebewertung hinausgehen müssten.

\section{Kapitel „Methoden der Nachhaltigkeit“}

Markus Berchtold-Domig berichtet über die „Entwicklung eines multivariablen Raumplanungsinstruments SHARC zur Verbesserung der inneren Stärke der Gemeinden in Vorarlberg gegenüber zukünftigen Anforderungen“; in einer Fallstudie konnte er die hervorgehobene Rolle von „Energie“ in der regionalen Entwicklung beobachten. $\mathrm{Ce}$ lina Martinez untersucht „Die Entwicklung städtebaulicher
Leitbilder im Metropolitanraum Zürich“. Sie betont deren Relevanz in partizipativen Prozessen und fordert mehr Forschung zu städtebaulichen Leitbildern in der Schweiz. Sandy-Cheril Manton versucht die „Ableitung von Indikatoren für ein nachhaltiges Campuskonzept im Bereich Energie- und Gebäudebewertung mithilfe geographischer Informationssysteme", welches, wenn in die Campusentwicklung integriert, eine nachhaltige Entwicklung der gesamten Universität verstärken könnte.

Insgesamt ist das Buch ein sehr empfehlenswertes zum Einlesen in aktuelle, durchaus normative, weil an Nachhaltigkeit orientierter Forschung aus der Stadt-, Regionalund Raumentwicklung. Insbesondere für diejenigen, die an Lösungsvorschlägen für die multiplen Krisen gesellschaftlicher Naturverhältnisse interessiert sind, finden sich in diesem Sammelband eine Vielzahl an Anregungen. 